\title{
INOVATIVIDADE E PERFORMANCES SOCIAL, AMBIENTAL E ECONÔMICA EM MPMES: UMA INVESTIGAÇÃO EMPÍRICA
}

\section{RESUMO}

Ações de inovação empresarial representam esforços para identificar estratégias e ações adaptativas e pró-ativa frente à complexidade do ambiente de negócios, a fim de melhorar a performance organizacional. Além disso, é necessário considerar a performance organizacional de acordo com as três dimensões de sustentabilidade (econômica, social e ambiental). Este estudo investiga as relações entre a inovatividade e as performances social, econômica e ambiental em indústrias de transformação do Rio Grande do Sul. A pesquisa foi realizada com a participação de 140 empresas. As relações hipotéticas do estudo foram estimadas por meio de análise de correlação e análise de regressão linear. Os resultados sugerem um impacto significativo e positivo da inovatividade nas performances ambiental, social e econômicas, com impacto mais acentuado sobre a dimensão ambiental. Também os resultados sugerem que existe uma inter-relação acentuada entre as três dimensões da performance organizacional sustentável.

Palavras-chave: Triple Botton Line; Inovatividade; Indústrias de Transformação; Performance Ambiental; Performance Social.

\section{INNOVATION AND SOCIAL, ENVIRONMENTAL AND ECONOMIC PERFORMANCES IN MSMES: AN EMPIRICAL APPROACH}

\begin{abstract}
Innovation actions represent business efforts to identify adaptive and proactive strategies and actions forward to the complexity of the business environment in order to improve organizational performance. Also, it is necessary to considerate the organizational performance based on the three dimensions of sustainability (economic, social and environmental). This study investigates the relationship between innovativeness and sustainable organizational performance (social, economic and environmental) in processing industries of Rio Grande do Sul. The survey was conducted with the participation of 140 companies. Hypothetical relations were investigated and estimated using correlation analysis and linear regression analysis. Results suggest the existence of a significant and positive impact of innovativeness on the environmental, social and economic performances, with a major impact on the environmental dimension. Also the results suggest that there is a strong interrelationship between the three dimensions of sustainable organizational performance.
\end{abstract}

Keywords: Triple Botton Line; Innovativeness; Processing Industries; Environmental Performance; Social Performance. 
Inovatividade e Performances Social, Ambiental e Econômica em MPMES: Uma Investigação Empírica

CARÁCTER INNOVADOR Y RENDIMIENTOS SOCIAL, AMBIENTAL Y ECONOMICO EN MIPYMES: UNA INVESTIGACIÓN EMPÍRICA

\section{RESUMEN}

Las acciones de innovación empresarial representan los esfuerzos para identificar estrategias y acciones adaptativas y proactivas frente la complejidad del entorno empresarial con el fin de mejorar el desempeño organizacional. Por otra parte, es necesario considerar el desempeño de la organización de acuerdo a las tres dimensiones de la sostenibilidad (económica, social y ambiental). Este estudio investiga la relación entre la innovación y los desempeños social, económico y ambiental en las industrias de transformación de lo estado de Rio Grande do Sul. La investigación se llevó a cabo con la participación de 140 empresas. Las relaciones hipotéticas del estudio se estimaron mediante el análisis de correlación y análisis de regresión lineal. Los resultados sugieren un impacto significativo y positivo de la innovación en los desempeños ambiental, social y económico, con un impacto más pronunciado en la dimensión ambiental. Además, los resultados sugieren que existe una fuerte interrelación entre las tres dimensiones del desempeño organizacional.

Palabras clave: Triple Botton Line; Carácter Innovador; Industrias Manufactureras; Desempeño Ambiental; Desempeño Social.

Eduardo Botti Abbade ${ }^{1}$

${ }^{1}$ Doutorando em Agronegócios pela Universidade Federal do Rio Grande do Sul - UFRGSS. Professor do Centro Universitário Franciscano e Analista Administrativo da Empresa Brasileira de Serviços Hospitalares (EBSERHHUSM). Brasil. E-mail: eduardo@unifra.br 


\section{INTRODUÇÃO}

As organizações, inseridas em um ambiente de alta competitividade e mudanças drásticas constantes, precisam rever e alterar suas posturas estratégicas de forma a atender às exigências dos mercados e a competir melhor frente a seus concorrentes. A partir dessa premissa do contexto atual dos negócios, uma postura empresarial com foco na adaptação às mudanças em prol da competitividade torna-se necessária. Essa postura de adaptabilidade às pressões ambientais e foco no desenvolvimento de estratégias de produtos e mercados é definida como postura inovativa ou inovatividade das empresas (Al-Tuwaijri, Christensen e Hughes, 2004; Rao e Holt, 2005). Nesse contexto, as organizações configuram-se como órgãos que fomentam a inovação em seus ambientes organizacionais, gerando melhorias estratégicas em seus produtos e processos.

As ações empresariais buscam constantemente aumentar a performance, a qual não é mais vista somente pelo viés econômico. As perspectivas sociais e ambientais de desempenho organizacional têm chamado a atenção de pesquisadores acadêmicos e de executivos tomadores de decisões estratégicas que percebem a importância de se atender às exigências e necessidades sociais e ambientais, uma vez que, possivelmente, essas performances influenciarão no desempenho econômico de seus negócios (Russo e Fouts, 1997; Miles e Covin, 2000; Al-Tuwaijri, Christensen e Hughes, 2004; Rao e Holt, 2005).

Este estudo tem como objetivo investigar as relações existentes entre as três dimensões da performance organizacional sustentável (social, econômica e ambiental) assim como a relação da inovatividade com tais dimensões da performance. Considerando que ações voltadas para a inovação organizacionais possam vir a impactar positivamente no desempenho organizacional, abrangendo a perspectiva do Triple Botton Line, este estudo contribuir com a construção do conhecimento científico acerca dessas relações de interdependência analisado sob a ótica de executivos de indústrias de transformação do Rio Grande do Sul. A consolidação de um entendimento teórico a respeito das relações existentes entre as dimensões do desenvolvimento sustentável poderá proporciona preencher algumas lacunas acadêmicas. Com isso, este estudo busca contribuir para o âmbito científico apresentando evidências que corroborem estudos já realizados. Ainda se considera como relevância prática deste estudo a contribuição gerada para o processo decisório, bem como o maior entendimento de como práticas estratégicas organizacionais voltadas para a inovatividade possam gerar elevados desempenhos organizacionais.

Esta pesquisa propõe relações hipotéticas a serem corroboradas por meio de evidências empíricas, com o intuito de contribuir para o campo científico de investigação organizacional, tendo como panorama as relações entre capacidades inovativas e desempenho organizacional. Por capacidades inovativas entende-se que são as capacidades de moldar e gerenciar capacidades múltiplas relacionadas à gestão de conhecimento, processos, tecnologia e inovação (Cetindamar et al., 2009). Com isso, a consolidação de uma compreensão maior acerca dos efeitos de orientações e posicionamentos estratégicos organizacionais sobre os desempenhos social, econômico e ambiental das organizações industriais é um dos focos centrais deste estudo. Além disso, a observação da realidade dessas organizações, no que tange à inovatividade, contribui para o processo decisório e elucida as preocupações atuais dos tomadores de decisão com foco não apenas em questões econômicas, como também em aspectos sociais e ambientais.

\section{INOVATIVIDADE E PERFORMANCE SUSTENTÁVEL ORGANIZACIONAL}

A literatura apresenta a inovação como um meio pelo qual as empresas podem se tornar mais competitivas. Uma abordagem baseada na inovação pode levar as organizações a uma posição diferenciada considerado o mercado em que atuam e os concorrentes. Siguaw, Simpson e Enz (2006) descrevem a abordagem da inovação como uma composição de filosofia de aprendizado, direcionamento estratégico e crenças da organização que definem as estratégias e ações específicas por meio de competências e processos inovadores. Os autores ainda acrescentam que a abordagem de inovação pode ser na alocação de recursos, tecnologias, funcionários, operações e mercados.

Observa-se que os resultados organizacionais e performance econômica podem ser relacionados à orientação para a inovação das organizações. Estudos apontam para a existência de uma relação significativa entre a inovação, aprendizagem organizacional e orientação para o mercado (Hurley e Hult, 1998; Perin, Sampaio e Hooley, 2007), a inovação e a orientação para o mercado (Webster e Gupta, 2005; Kohli e Jaworski, 1990), inovação, orientação para o mercado e orientação para o empreendedorismo (Baker e Sinkula, 2009) e orientação de aprendizagem, orientação para o mercado e inovação (Baker e Sinkula, 1999).

A inovação está fortemente relacionada com a capacidade organizacional de inovar, o que significa uma abertura da organização a novas ideias (Hurley e Hult, 1998). Tal competência é um aspecto da cultura organizacional, cuja ênfase está na aprendizagem, na participação na tomada de decisões, no apoio, na colaboração e no poder compartilhado. Todos estes elementos, em conjunto ou de forma independente, podem afetar a orientação para a inovação. Adicionalmente, a capacidade de inovação se refere às 
Inovatividade e Performances Social, Ambiental e Econômica em MPMES: Uma Investigação Empírica

ações focadas em posturas adaptativas e à adoção de novas idéias, processos e produtos com sucesso. É digno de nota que a orientação para o mercado, a orientação para a aprendizagem e a inovação organizacional são as propriedades que afetam o processo de inovação. Assim, tanto a inovação efetiva quanto a capacidade de inovar resultam em vantagens competitivas e aumento do desempenho organizacional (Hurley e Hult, 1998; Hult, Hurley e Knight, 2004).

Da mesma forma, o estudo de Zhou, Yim e Tse (2005) destaca-se pelo design e teste de um modelo com diferentes tipos de forças estratégicas e de mercado, incluindo a aprendizagem organizacional, a inovação e o desempenho organizacional. Os autores concluíram que as forças de mercado (incerteza da demanda, a turbulência, tecnologia e intensidade competitiva) exercem uma influência significativa sobre inovações tecnológicas e inovações com base no mercado, e estes dois tipos de inovações afetam o desempenho organizacional de formas distintas.

Inicialmente é necessário considerar que o desempenho orgaizacional não está restrito apenas à dimensão econômica. A performance sustentável organizacional está baseada nas três dimensões do conceito central da sistentabilidade - triple botton line - que são as performances econômica, social e ambiental. Dais orientações empresariais foram fundamentadas no desenvolvimento e amadurecimento conceitual da sustentabilidade e desenvolvimento sustentável.

A partir dos anos de 1990, o termo desenvolvimento sustentável (DS) passou a receber maior destaque na academia onde alguns pontos de discussão são frequentemente observados como a perspectiva de análise da pegada ecológica (ecological footprint) (Van den Bergh e Verbruggen 1999; Gottlieb et al. 2012), a responsabilidade social corporativa (Carroll, 1999) e a abordagem do Triple Botton Line (Elkington, 1998). O foco da analise do desempenho organizacional no presente estudo é centrado nas três dimensões do Triple Botton Line: econômica ou produtiva, ambiental e social.

A preocupação ambiental iniciou, principalmente, com a Conferência de Estocolmo na Suécia em 1972. A principal definição do conceito de desenvolvimento sustentável partiu do Relatório de Brundtland, em que se define desenvolvimento sustentável como aquele "[...] desenvolvimento que atenda às necessidades do presente sem comprometer a capacidade das gerações futuras satisfazerem suas próprias necessidades" (WCDE, 1987, p. 23, tradução nossa) Um maior alcance e reconhecimento foi possível com a Conferência da Organização das Nações Unidas sobre Meio Ambiente e Desenvolvimento realizada no Rio de Janeiro e com a criação da Agenda 21, em que se destaca a importância de um desenvolvimento local com vistas para o global.

Nos últimos anos, a discussão sobre o conceito de desenvolvimento sustentável surgiu como um esforço para resolver os problemas ambientais causados pelo processo de produção e crescimento econômico, sendo um termo considerado como uma das principais palavras de ordem nos dias atuais (Banerjee, 2003). O progresso e modernidade observados nas últimas décadas trouxeram muitos benefícios. No entanto, alguns danos foram gerados para o planeta, como a destruição da camada de ozônio, a redução da biodiversidade, o aquecimento global, a poluição do ar e da água, entre outros (Paulraj, 2011). Configura-se assim a necessidade de um olhar advindo das três principais dimensões do desenvolvimento sustentável.

Associa-se a dimensão econômica ao crescimento produtivo. Logo, essa dimensão pode ser avaliada por meio da análise dos rendimentos, lucratividade, despesas, níveis de emprego, fatores de negócios, participação de mercado etc.. A dimensão social pode ser analisada por meio de variáveis como educação, acesso a serviços sociais, saúde, bem-estar, capital social e qualidade de vida da população. A dimensão ambiental se refere, em síntese, na compreensão de variáveis como medições do gás natural, qualidade da água e do ar, consumo de energia e recursos naturais, resíduos tóxicos e sólidos gerados pelo homem e pelo processo produtivo (Slaper e Hall, 2011).

Em geral, estudos sugerem a existência de uma relação positiva entre a inovação e o desempenho econômico das empresas. No entanto, é necessário destacar a relação da inovação com os demais elementos do desempenho organizacional direcionados para a sustentabilidade. Por exemplo, a relação entre a capacidade de inovação e o desempenho ambiental tem sido estudada por Russo e Fouts (1997). Os autores entrevistaram 243 empresas e concluiram que para as empresas "vale a pena ser verde", isto é, o desenvolvimento ambiental fortalece o crescimento da indústria. Já Rao e Holt (2005) investigaram a relação entre as práticas de cadeias de suprimento verdes, o desempenho econômico, o desempenho ambiental e a competitividade em uma amostra de empresas do Sudeste Asiático. Os resultados indicaram que as empresas estavam em diferentes fases da cadeia de suprimentos verde, mas que isso poderia levar a um aumento da competitividade e do desempenho econômico. Adicionalmente, evidencias sugerem que uma identidade organizacional verde influencia positivamente o desempenho da inovação verde, principalmente em empresas de grande porte (ChingHsun e Yu-Shan, 2013). Ainda considerando tal relação, evidencias indicam que práticas de inovação ambientais impactam e forma positiva e significativa na performance ambiental das empresas, enquanto que o efeito sobre a performance financeira se dá por intermédio da performance ambiental (Li, 2014).

Já o desempenho social é destacado no estudo de Menon e Menon (1997), que considera a relação entre o desempenho social, orientação empreendedora 
e estratégia de marketing através da integração de preocupações ambientais nas políticas de desenvolvimento e práticas de marketing. Logo, a inovação associada aos processos de marketing pode vir a impactar positivamente na performance social das organizações. Apesar dos poucos estudos associando diretamente o constructo de inovação com a performance social organizacional, evidências sugerem que investimentos em Responsabilidade Social Coorporativa são interpretados por agentes de crédito como um indicador de desempenho financeiro elevado das empresas (Guiral, 2012). Outro estudo suporta a premissa de que inovações tecnológicas e organizacionais estão mais relacionadas ao desempenho econômico, enquanto inovações de produtos possuem um maior impacto sobre o desempenho social das organizações (Garrido e Camarero, 2010).

\section{RELAÇÕES HIPOTÉTICAS DO ESTUDO}

Baseado no que foi exposto anteriormente, e considerando o objetivo do presente estudo que é investigar as relações existentes entre as três dimensões da performance organizacional sustentável (social, econômica e ambiental) assim como a relação da inovatividade com tais dimensões da performance, foram definidas 6 hipóteses a serem testadas empiricamente.

Um dos pontos centrais do modelo proposto por Carter e Rogers (2008) é de que apesar de possíveis resultados positivos e satisfatórios alcançados por meio da integração limitada das performances organizacionais, a verdadeira performance superior e sustentável corporativa será alcançada mediante a integração das três dimensões da sustentabilidade propostas por Elkington (1998). Estudos relacionados à sustentabilidade e a preocupação coorporativa com aspectos ambientais e sociais têm emergido com grande força nos últimos anos de modo a abordar questões ambientais importantes (Elkington, 1998; Newell e Muro, 2006; Schaefer, 2004; Wagner, 2005). Evidências de estudos anteriores já sugerem a existência de uma associação positiva entre a performance social e econômica (Orlitzky, Schmidt e Rynes, 2003; Guiral, 2012) assim como da performance ambiental e econômica (Wagner, 2005; Russo e Fouts, 1997; Rao e Holt, 2005) e da performance social e ambiental (Schaefer, 2004). Logo, tais embasamentos dão sustentação para as três primeiras hipóteses deste estudo.

Hipótese 1: A performance econômica está positivamente e significativamente correlacionada à performance ambiental.

Hipótese 2: A performance econômica está positivamente e significativamente correlacionada à performance social.
Hipótese 3: A performance ambiental está positivamente e significativamente correlacionada à performance social.

Estudos evidenciam que a inovatividade resulta em obtenção de vantage competitive e aumento de performance organizacional (Hurley e Hult, 1998; Baker e Sinkula, 1999; Hult, Hurley e Knight, 2004; Zhou, Yim e Tse, 2005). No entanto, a performance organizacional pode ser desdobrada nas três dimensões da performance sustentável, e poucas evidências estão disponíveis considerando tais dimensões da performance. A inovatividade, sendo considerada como a capacidade de inovar e que tende a resultar em vantagens competitivas e aumento do desempenho organizacional (Hurley e Hult, 1998; Hult, Hurley e Knight, 2004), possivelmente impacte positivamente no desenvimento de iniciativas, processos e produtos relacionados com a performance ambiental empresarial. Estudos já evidenciam a associação positiva entre a inovatividade e a performance ambiental (Russo e Fouts, 1997) salientando que "vale a pena ser verde". Logo, tais argumentos dão sustentação à hipótese 4 deste estudo.

Hipótese 4: A inovatividade empresarial econômica impacta positivamente e significativamente na performance ambiental.

Já, considerndo a possível relação positiva entre a inovatividade e a performance social, poucas evidências são encontradas para dar sustentação a tal argumento. Mas é razoavel supor que ações inovativas em processos e estratégias organizacionais podem fomentar uma melhora no relacionamento social da organização com seus divesos stakeholders. Tendo isso em mente, estudo evidencia que a inovatividade atua como mediador da orientação para o mercado, orientação para a aprendizagem e a responsabilidade coorporativa, fomentando a performance organizacional e social (Han et al., 2013). Tal argumento embasa a hipótese 5 deste estudo.

Hipótese 5: A inovatividade empresarial econômica impacta positivamente e significativamente na performance social.

Retomando o conceito já apresentado de inovatividade, este estudo parte do argumento de que a inovatividade de uma empresa facilita o desenvolvimento de novos produtos e processos organizacionais contribuindo para o desempenho organizacional (Calantone, Garcia e Droge, 2003; Droge, Calantone e Harmancioglu, 2008). Considerando a relação entre a inovatividade empresarial e a performance econômica, evidências sugerem que a existência empíricas argumentam para a existência de tal associação positiva (Tsai e Yang, 2012; Gualandris e Kalchschmidt, 2014). Considerando os argumentos anteriormente apresentados, foi elaborada a última hipótese deste estudo (hipótese 6) a ser testada empiricamente. 
Hipótese 6: A inovatividade empresarial econômica impacta positivamente e significativamente na performance econômica.

A figura 1 ilustra essas relações a serem estimadas para as indústrias de transformação do Rio Grande do Sul. As hipóteses H1, H2 e H3 sumarizam a existência de correlação positiva e significativa entre as dimensões da performance organizacional sustentável. Já as hipóteses H4, H5 e H6 assumem que a inovatividade impacta positivamente e significativamente nas performances ambiental, social e econômica respectivamente.

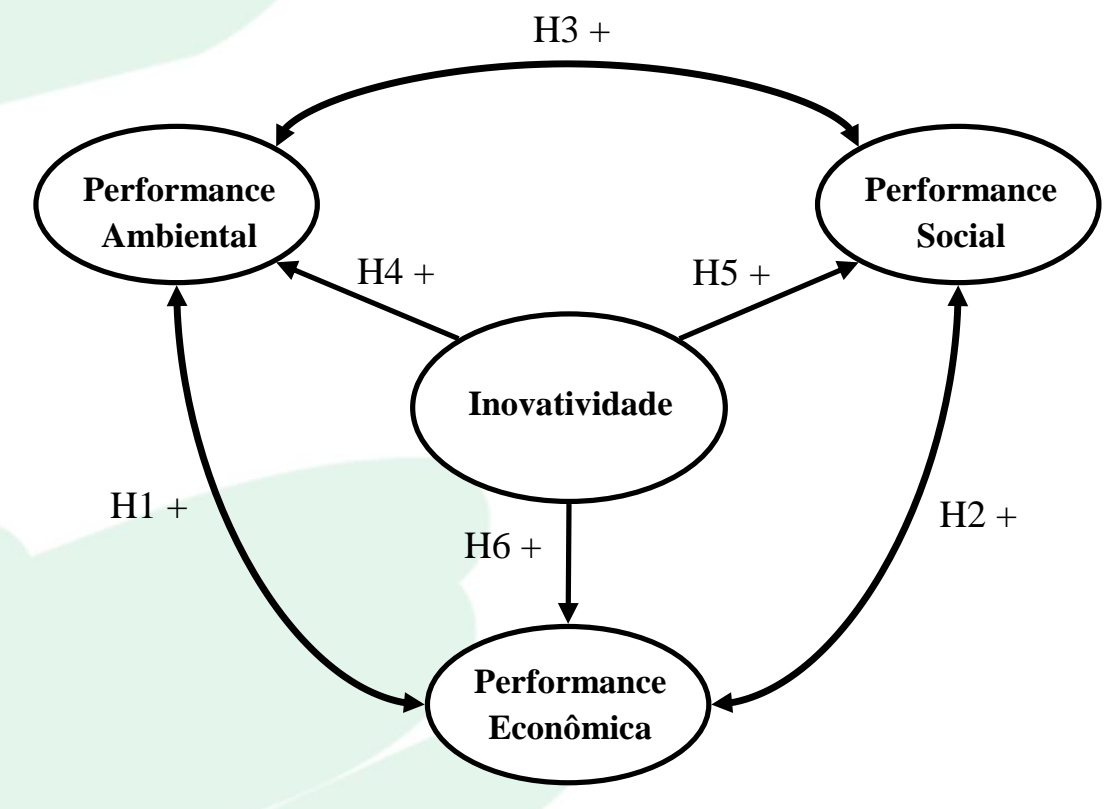

Figura 1 - Relações hipotéticas entre as variáveis do estudo

Em suma, infere-se que o desempenho financeiro das organizações pesquisadas é influenciada pelo desempenho ambiental e social. No entanto, a relação causal é altamente complexa de ser investigada. Com isso, este estudo busca analisar a correlação existente entre as dimensões da performance organizacional sustentável. Estudos sugerem que as organizações que incorporam ações ambientais em suas práticas organizacionais ter um resultado financeiro melhor do que aqueles que não tomar essa decisão (Russo e Fouts, 1997; Miles e Covin, 2000; AlTuwaijri, Christensen e Hughes, 2004; Rao e Holt, 2005). O mesmo ocorre com as pesquisas que relacionam a adoção de práticas organizacionais sociais com melhores resultados financeiros (Knack e Keefer, 1997; Orlitzky, Schmidt e Rynes, 2003). Adicionalmente, evidências sugerem que a inovatividade impacta positivamente na performance organizacional (Baker e Sinkula, 1999).

\section{PROCEDIMENTOS METODOLÓGICOS}

Este estudo se caracteriza por ser um survey elaborado por meio de um levantamento único com corte transversal. Os procedimentos foram adotados de acordo com o delineamento descritivo. Detalhes a respeito das etapas e procedimentos metodológicos de elaboração, coleta e análise de dados são apresentados a seguir.

\subsection{Intrumento e Coleta de Dados}

O instrumento de coleta de dados foi elaborado com base em escalas e índices já testados, validados e utilizados em outros estudos. Tais escalas, originalmente desenvolvidas em inglês, foram traduzidas com o acompanhamento técnico de profissional lingüista, passando posteriormente pela avaliação de um pesquisador da área do estudo e por um empresário habilitado. $\mathrm{O}$ primeiro constructo que compõe o instrumento de coleta de dados é a escala de avaliação a Inovatividade das MPMEs pesquisadas e foi elaborado com base na escala de avaliação elaborada por Hurley e Hult (1998). Essa escala foi composta por cinco afirmações que foram avaliadas por meio de uma escala Likert de sete pontos. Os itens que avaliam o desempenho das empresas pesquisadas são distribuídos em três blocos de questões de acordo com o modelo do Triple Botton Line. Cada um dos 
desempenhos Social, Econômico e Ambiental foram avaliados por meio de 5 afirmativas, originalmente desenvolvidas por Carter e Rogers (2008) e obtidas em artigo publicado por Paulraj (2011). As afirmações que compuseram o inventário de avaliação dos três desempenhos organizacionais foram avaliadas por meio de uma escala Likert de sete pontos.

O instrumento de coleta de dados foi elaborado de forma eletrônica onde o formulário online foi hospedado e disponibilizado para os respondentes em um endereço eletrônico. O link de acesso ao instrumento foi enviado para 4580 indústrias de transformação atuantes no estado do Rio Grande do Sul / Brasil. O cadastro dos e-mails das empresas foi obtido no Cadastro da Indústria da FIERGS de 2010. Foi enviado juntamente ao e-mail um texto de apresentação do estudo. Os e-mails com o texto de apresentação e o link de acesso ao instrumento foram reenviados mais duas vezes para as organizações cadastradas, com um intervalo de uma semana, totalizando três semanas de coleta de dados. Ao final do período de coleta de dados, o retorno obtido foi de 140 questionários considerados válidos e oriundos de MPMEs de segmentos industriais diversos.

\subsection{Procedimentos de Análise de Dados}

$\mathrm{Na}$ análise dos dados inicialmente foram observados os resultados obtidos para as variáveis adotadas para avaliar o tamanho das empresas que aderiram ao estudo (receita bruta anual e número de colaboradores). Foram selecionadas apenas as empresas classificadas como micro, pequenas e médias empresas que totalizaram 140 empresas que aderiram ao estudo. Estas também foram classificadas de acordo com o tido de atividade industrial que desempenham.

Para avaliar a validade e eficiência dos constructos deste estudo foram observados alguns índices e valores de mensuração obtidos com auxílio do software SPSS 14. O AVE (Variância Média Extraída) representa a avaliação da validade convergente e deve apresentar valores superiores a 0,5. A Confiabilidade Composta mensura a precisão com a qual a variável latente mede aquilo que se propõe a medir deve apresentar valores superiores a 0,7 (Fornell e Larcker, 1982). A Confiabilidade Composta, e o AVE são calculados a partir das cargas padronizadas das variáveis latentes e dos erros obtidos na análise fatorial (Hair et al., 1998). Também foi analisado o valor do coeficiente KMO (Kaiser-Meyer-Olkin) que indica a proporção da variância dos dados que pode ser considerada comum aos indicadores. Da mesma forma que o AVE, sugerese que o KMO deva apresentar valor superior a 0,5 (Hair et al., 1998). Observou-se também o valor do Alfa de Cronbach, que determina a validade interna do constructo, considerando-se valores acima de 0,7 como satisfatórios.

A análise das relações hipotéticas entre as variáveis do estudo foram investigadas por meio de análise de correlação de Pearson e analise de regressão linear simples. De modo a avaliar a validade das equações de regressão, foram calculados e avaliados os valores de $\mathrm{R}^{2}$ ajustado (coeficiente de determinação) e a significância das equações ( $\mathrm{p}$-valor). $\mathrm{O}$ valor de $\mathrm{R}^{2}$ (coeficiente de determinação) é o valor índice que indica a percentagem da variância total da variável dependente que é explicada pela equação de regressão (Hair et al., 1998). Os resultados dos procedimentos estatísticos e analíticos são descritos na próxima seção.

\section{RESULTADOS}

Nesta seção são apresentados os resultados obtidos no estudo assim como as interpretações analíticas. Na primeira parte, são apresentados os resultados dos dados obtidos referentes ao tamanho das organizações (receita bruta anual e número de funcionários). Já na segunda parte destacam-se os resultados obtidos para as variáveis centrais do estudo e a natureza das relações entre as mesmas.

\subsection{Descrição da Amostra Estudada}

Ao pesquisar as indústrias de transformação do Rio Grande do Sul, obteve-se a adesão voluntária de 140 empresas de micro, pequeno e médio porte. O quadro 1 abaixo apresenta a classificação das empresas quanto as suas atividades assim como a quantidade de respostas obtidas em cada segmento de atuação industrial. Também é disponibilizado o total de empresas do Rio Grande do Sul nos segmentos observados (IBGE, 2009). 
Inovatividade e Performances Social, Ambiental e Econômica em MPMES: Uma Investigação Empírica

\begin{tabular}{|c|c|c|}
\hline RAMO DE ATUAÇÃO & $\begin{array}{l}\text { Empresas da } \\
\text { amostra }\end{array}$ & $\begin{array}{l}\text { Empresas no } \\
\text { RS }\end{array}$ \\
\hline Alimentos e Bebidas & 35 & 6.743 \\
\hline Metalurgia, máquinas e equipamentos, produtos de metal e veículos automotores & 33 & 10.792 \\
\hline $\begin{array}{l}\text { Couros, fabricação de artefatos de couro, produtos têxteis, artigos de vestiário e } \\
\text { acessórios }\end{array}$ & 19 & 12.284 \\
\hline Produtos de borracha e de material plástico & 18 & 1.896 \\
\hline Móveis e produtos de madeira & 11 & 7.012 \\
\hline Produtos diversos e manutenções. & 7 & 3.830 \\
\hline Produtos de minerais não-metálicos & 6 & 2.552 \\
\hline $\begin{array}{l}\text { Equipamentos de informática, produtos eletrônicos e ópticos, aparelhos e materiais } \\
\text { elétricos }\end{array}$ & 6 & 1.072 \\
\hline Produtos químicos & 5 & 999 \\
\hline TOTAL & 140 & 47.180 \\
\hline
\end{tabular}

Quadro 1 - Empresas pesquisadas quanto ao ramo de atuação industrial

É possível constatar que as indústrias de alimentos, bebidas juntamente com as indústrias pesadas (metalurgia, máquinas e equipamentos, produtos de metal e veículos automotores) representam cerca de $50 \%$ da amostra obtida. Os demais segmentos industriais apresentaram menor retorno. É necessário salientar, no entanto, que uma limitação importante do estudo é baixa adesão ao estudo por parte dos empresários. Apesar do esforço realizado e do envio do formulário eletrônico para 4.580 empresas com e-mails disponibilizados no Cadastro Industrial da FIERGS (2010), a baixa adesão pode representar um desinteresse, e possivelmente uma falta de comprometimento, dos executivos com esse tipo de investigação científica.

Seguindo na classificação das empresas que aderiram ao estudo, são apresentadas as distribuições de acordo com a receita bruta anual e a quantidade de funcionários. A classificação do tamanho das empresas é disponibilizada pelo Banco Nacional de Desenvolvimento Econômico e Social (BNDES, 2010) e está de acordo com a resolução do ano de 2010. Quanto ao número de colaboradores, utilizou-se a classificação do Serviço Brasileiro de Apoio às Micro e Pequenas Empresas (SEBRAE, 2011).

Tabela 1 - Empresas pesquisadas de acordo com a receita bruta anual e o número de funcionários.

\begin{tabular}{|c|c|c|c|}
\hline VARIÁVEL & CLASSIFICAÇÃO DE PORTE & FREQUÊNCIA & PORCENTAGEM \\
\hline \multirow{5}{*}{$\begin{array}{c}\text { Receita } \\
\text { bruta anual }\end{array}$} & até $\mathrm{R}$ \$2,4 milhões (Microempresa) & 76 & $54,29 \%$ \\
\hline & entre $\mathrm{R} \$ 2,4$ milhões e $\mathrm{R} \$ 16$ milhões (Pequena empresa) & 32 & $22,86 \%$ \\
\hline & entre $R \$ 16$ milhões e $R \$ 90$ milhões (Média empresa) & 18 & $12,86 \%$ \\
\hline & entre $\mathrm{R} \$ 90$ milhões e $\mathrm{R} \$ 300$ milhões (Média-grande empresa) & 9 & $6,43 \%$ \\
\hline & sem resposta & 5 & $3,57 \%$ \\
\hline \multirow{5}{*}{$\begin{array}{l}\text { Número de } \\
\text { funcionários }\end{array}$} & até 19 funcionários (Microempresa). & 66 & $47,14 \%$ \\
\hline & de 20 até 99 funcionários (Pequena empresa). & 47 & $33,57 \%$ \\
\hline & de 100 até 499 funcionários (Média empresa). & 18 & $12,86 \%$ \\
\hline & mais de 500 (Grande empresa). & 7 & $5,00 \%$ \\
\hline & sem resposta & 2 & $1,43 \%$ \\
\hline TOTAL & & 140 & $100 \%$ \\
\hline
\end{tabular}

Por meio da análise da distribuição das empresas quanto a receita bruta anual e número de funcionários foi observado que todas as empresas analisadas são classificadas como MPMEs. Analisando os dois critérios de forma conjunta, todas as 140 empresas podem ser consideradas como MPMEs já que nenhuma das respostas obtidas apresenta a classificação de grande empresa nos dois critérios observados.

\subsection{Análises Relacionais}

Nesta subseção da análise dos resultados do estudo, são analisadas as estatísticas descritivas (média aritmética geral e desvio-padrão geral) de cada um dos constructos do modelo de referência, que são a Inovatividade, o Desempenho Ambiental, o Desempenho Social e o Desempenho Econômico. Também são abordados os resultados da análise fatorial 
onde são expostos os valores das cargas fatoriais de cada um dos itens que compuseram os constructos do modelo de referência assim como os valores do alfa de
Cronbach, da confiabilidade composta e do AVE de cada constructo (variáveis latentes). Na Tabela 2, são apresentados os valores citados.

Tabela 2 - Estatísticas descritivas dos itens do estudo

\begin{tabular}{|c|c|c|c|c|c|c|c|c|}
\hline & & $\begin{array}{l}\text { Média } \\
\text { global }\end{array}$ & $\begin{array}{c}\text { Desvio } \\
\text { padrão } \\
\text { global }\end{array}$ & $\begin{array}{l}\text { Carga } \\
\text { fatorial }\end{array}$ & $\begin{array}{c}\text { Alfa de } \\
\text { Cronbach }\end{array}$ & $\begin{array}{c}\text { Confiabilidade } \\
\text { composta }\end{array}$ & KMO & AVE \\
\hline \multirow{5}{*}{$\begin{array}{l}\text { Performance } \\
\text { Ambiental }\end{array}$} & DA1 & \multirow{5}{*}{5,906} & \multirow{5}{*}{1,313} & $0,826 *$ & \multirow{5}{*}{0,873} & \multirow{5}{*}{0,908} & \multirow{5}{*}{0,842} & \multirow{5}{*}{0,663} \\
\hline & DA2 & & & $0,860 *$ & & & & \\
\hline & DA3 & & & $0,823 *$ & & & & \\
\hline & DA4 & & & $0,827 *$ & & & & \\
\hline & DA5 & & & $0,731 *$ & & & & \\
\hline \multirow{5}{*}{$\begin{array}{l}\text { Performance } \\
\text { Econômica }\end{array}$} & DE1 & \multirow{5}{*}{5,547} & \multirow{5}{*}{1,211} & $0,623 *$ & \multirow{5}{*}{0,779} & \multirow{5}{*}{0,848} & \multirow{5}{*}{0,713} & \multirow{5}{*}{0,529} \\
\hline & DE2 & & & $0,818 *$ & & & & \\
\hline & DE3 & & & $0,700 *$ & & & & \\
\hline & DE4 & & & $0,791 *$ & & & & \\
\hline & DE5 & & & $0,688 *$ & & & & \\
\hline \multirow{5}{*}{$\begin{array}{l}\text { Performance } \\
\text { Social }\end{array}$} & DS1 & \multirow{5}{*}{5,699} & \multirow{5}{*}{1,167} & $0,715 *$ & \multirow{5}{*}{0,861} & \multirow{5}{*}{0,900} & \multirow{5}{*}{0,831} & \multirow{5}{*}{0,645} \\
\hline & DS2 & & & $0,808 *$ & & & & \\
\hline & DS3 & & & $0,831 *$ & & & & \\
\hline & DS4 & & & $0,782 *$ & & & & \\
\hline & DS5 & & & $0,871 *$ & & & & \\
\hline \multirow{3}{*}{ Inovatividade } & INO1 & & & $0,757 *$ & \multirow{3}{*}{0,766} & \multirow{3}{*}{0,867} & \multirow{3}{*}{0,648} & \multirow{3}{*}{0,686} \\
\hline & INO2 & 4,843 & 1,499 & $0,888 *$ & & & & \\
\hline & INO3 & & & $0,835 *$ & & & & \\
\hline
\end{tabular}

A partir da observação dos valores das médias e desvios padrões constata-se que a Inovatividade nas empresas pesquisadas é considerada como sendo moderadamente positiva apresentando também um valor de dispersão relativamente acentuado. Quanto às variáveis que avaliam o desempenho organizacional (social, econômico e ambiental) considera-se que as empresas pesquisadas atestam ter elevados desempenhos nas três perspectivas de análise avaliadas assim com moderadas dispersões entre as respostas obtidas.

Referente aos valores das cargas fatoriais obtidas na análise fatorial dos indicadores de cada constructo observa-se que todos os itens dos constructos apresentam significância ao comporem as respectivas variáveis latentes. Apesar de serem observados alguns itens com cargas fatoriais baixas (ex. item DE1), estes foram mantidos de modo a preservar as escalas originais assim como os atributos teóricos dos itens. Entretanto, dois itens foram excluídos da escala de avaliação de Inovatividade por não terem atingido valores satisfatórios de carga fatorial.

Ao analisar os valores do Alfa de Cronbach constata-se que todos os valores são superiores a 0,7, o que permite afirmar que os constructos possuem uma satisfatória validade interna. O mesmo ocorre quando se analisa os valores da Confiabilidade composta os quais também são todos superiores a 0,7. Os valores obtidos para os AVEs dos constructos são também considerados satisfatórios já que são todos superiores a 0,5 (representando mais da metade da variância dos itens dos constructos). Adicionalmente, os valores do KMO são também altamente satisfatórios o que sugere que a amostra do estudo é adequada.

A seguis são observados na tabela 3 os valores das correlações obtidas entre os constructos do estudo assim como os valores da raiz quadrada dos AVEs na diagonal principal expostos em negrito. Vale ressaltar é aconselhado que esses últimos valores citados sejam superiores aos valores das correlações com as demais variáveis latentes.

Tabela 3 - Correlação entre as variáveis latentes e raiz quadrada do AVE (diagonal)

\begin{tabular}{|l|c|c|c|c|}
\hline & $\mathbf{1 .}$ & $\mathbf{2 .}$ & $\mathbf{3 .}$ & $\mathbf{4 .}$ \\
\hline 1. Performance Ambiental & $\mathbf{0 , 8 1 4}$ & & & \\
\hline 2. Performance Econômica & $0,451^{* *}$ & $\mathbf{0 , 7 2 7}$ & & \\
\hline 3. Performance Social & $0,713^{* * *}$ & $0,503^{* * *}$ & $\mathbf{0 , 8 0 3}$ & \\
\hline 4. Inovatividade & $0,224 * *$ & $0,198^{*}$ & $0,182^{*}$ & $\mathbf{0 , 8 2 8}$ \\
\hline
\end{tabular}

$$
\text { Nota: } * \mathrm{p}<0,05 ; * * \mathrm{p}<0,01 ; * * * \mathrm{p}<0,001
$$


Observando os valores das correlações constata-se que os desempenhos organizacionais (econômico, social e ambiental) são fortemente correlacionados sugerindo uma forte relação entre os mesmos. Logicamente não é possível afirmar que existe uma relação de interdependência simplesmente por meio da observação dos valores das correlações. Entretanto, essa constatação já permite sugerir que os desempenhos organizacionais baseados no triple botton line são inter-relacionados. Também se constata que os desempenhos ambiental, econômico e social estão significativamente correlacionados à inovatividade das empresas pesquisadas, o que permite afirmar que a postura de encorajamento à inovação das mesmas está atrelada ao desempenho organizacional sustentável.

Dando continuidade nas análises dos dados coletados, foram realizadas análises de regressão linear com o intuito de verificar a influência da inovatividade em cada uma das dimensões da performance organizacional sustentável. Os resultados das três relações investigadas são apresentados na tabela 4 .

Tabela 4 - Análise de regressão linear para as hipóteses de estudo

\begin{tabular}{|c|c|c|c|c|c|c|}
\hline \multirow{2}{*}{$\begin{array}{l}\text { Variável independente } \\
\text { Variáveis dependentes }\end{array}$} & \multicolumn{2}{|l|}{ Constante } & \multicolumn{2}{|c|}{ Inovatividade } & \multirow{2}{*}{$\mathbf{R}^{2}$ ajustado } & \multirow{2}{*}{ p-valor } \\
\hline & $\mathrm{B}$ & p-valor & $\mathrm{B}$ & p-valor & & \\
\hline Performance Ambiental & $1,01 \mathrm{E}-16$ & 1 & 0,224 & $0,007 * *$ & 0,044 & $0,007 * *$ \\
\hline Performance Econômica & $1,78 \mathrm{E}-16$ & 1 & 0,198 & $0,018 *$ & 0,033 & $0,018 *$ \\
\hline Performance Social & $-4,9 \mathrm{E}-17$ & 1 & 0,182 & $0,031 *$ & 0,026 & $0,031 *$ \\
\hline
\end{tabular}

Nota: $* \mathrm{p}<0,05 ; * * \mathrm{p}<0,01 ; * * * \mathrm{p}<0,001$

Por meio da análise de regressão linear é possível constatar que a inovatividade impacta de forma positiva e significativa nas três dimensões da performance organizacional sustentável das industrias de transformação gaúchas. Ainda é possível considerar que a inovatividade das indústrias pesquisadas apresenta um impacto mais acentuado sobre a performance ambiental, o que sugere que as ações inovadoras das industrias gaúchas estão mais voltadas para questões e preocupações ambientais. A figura 2 apresenta os resultados do estudo de forma esquemática.

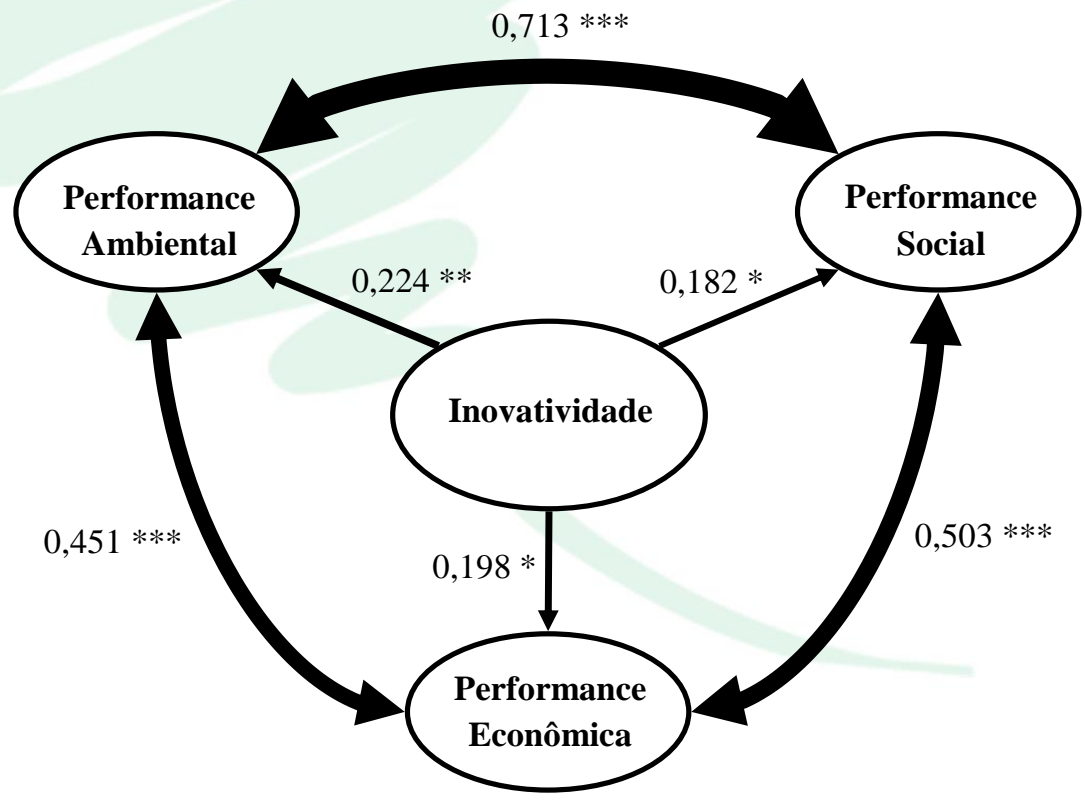

Figura 2 - Resultados da avaliação das relações do estudo. Nota: * $\mathrm{p}<0,05 ; * * \mathrm{p}<0,01 ; * * * \mathrm{p}<0,001$.

Os resultados do presente estudo sugerem que existe uma correlação acentuada e positiva entre as dimensões da performance organizacional sustentável. Além disso, é possível observar que a correlação existente entre as dimensões ambiental e social é extremamente elevada, o que sugere que tais dimensões estão mais fortemente inter-relacionadas. Além disso, como já foi exposto, a inovatividade impacta positivamente nas três dimensões da performance organizacional sustentável, 
com destaque para o impacto da inovatividade na performance ambiental.

\section{CONCLUSÕES}

Determinadas ações e posturas estratégias empresariais são constantes objetos de estudos acadêmicos que buscam relacioná-las ao desempenho organizacional. Entretanto, observa-se que as empresas buscam, além da melhoria do desempenho econômico, também elevação em suas performances ambientais e sociais. Sendo esses três pilares (econômico, social e ambiental) que sustentam as premissas do desenvolvimento sustentável, as empresas devem delinear suas posturas estratégicas de modo a balancear esses três desempenhos. Ainda observa-se que o desempenho prioritário é o econômico, mas já se constata a preocupação de certas organizações em manter uma performance social e ambiental superiores de modo a atingir melhores performances econômicas, mesmo que poucos estudos relatem essas constatações com clareza.

Tendo em vista que a as relações entre as dimensões econômica, ambiental e social do desempenho organizacional não são explorada amplamente em estudos de cunho acadêmico, este estudo teve como objetivo investigar as relações existentes entre a Inovação e os Desempenhos Social, Econômico e Ambiental. As organizações investigadas foram MPMEs atuantes em diversos setores industriais de transformação localizadas no Rio Grande do Sul (Brasil). Essas empresas demonstraram ter uma Inovatividade medianamente desenvolvida. Também foi constatado que as empresas demonstram ter elevados desempenhos econômico, social e ambiental. Entretanto vale ressaltar que essa avaliação do desempenho é baseada na percepção dos respondentes, uma vez que a mensuração e a avaliação foram efetuadas por meio de escala estruturada subjetiva e não baseadas em indicadores econômicos e contábeis.

Quanto aos resultados obtidos para as relações hipotéticas do estudo, foi constatado que a Inovatividade das empresas impacta significativamente nas dimensões Ambiental, Econômica e Social do desempenho sustentável das empresas. Isso sugere que a postura de inovatividade das empresas resulta em desempenhos superiores relativos a aspectos ambientais (como redução de emissões, economia de energia e minimização de impactos ecológicos negativos), aspectos sociais (bem estar da comunidade, qualidade de vida de colaboradores e justiça social) e aspectos econômicos (lucratividade, retorno financeiro, redução de custo e receitas). Salienta-se ainda que a inovatividade impacta de forma mais acentuada sobre a dimensão ambiental da performance organizacional, sugerindo que as ações inovadoras das industrias gaúchas estão mais voltadas para preocupações ambientais. Além disso, as evidências também sugerem que existe uma inter-relação bastante forte entre as três dimensões da performance organizacional sustentável. Com isso, a construção de uma performance organizacional superior deve se dar por meio de um balanceamento entre as dimensões social, econômica e ambiental.

Ressalta-se aqui a importância da realização de estudos adicionais que consolidem os aspectos teóricos e conceituais a respeito das interrelações existentes entre os desempenhos ambiental, econômico e social. A proximidade e inter-relação dessas performances levantam questionamentos importantes quanto à natureza dos mesmos e seus níveis de abrangência. Considerando os aspectos ambientais e sociais, pode-se indagar que o desempenho ambiental apresenta um foco maior em ações que impactam de forma positiva no âmbito externo das organizações. Com isso, seus resultados podem tender a serem revertidos em desempenho econômico ao longo prazo. Já o desempenho social pode ser determinado por ações com foco interno e externo efetuadas pelas organizações, considerando o bem-estar do público interno (colaboradores) e externo (comunidade na qual estão inseridas). Supõe-se ainda que ações em prol do desempenho social possam vir a impactar o desempenho econômico em menor prazo, quando comparadas às ações em prol do meio ambiente. Além disso, ações decorrentes de preocupações ambientais, como redução de emissões de gases do efeito estufa e lixos tóxicos, apresentam invariavelmente impactos ambientais e sociais. Assim, entende-se que é necessário delinear de forma mais clara as fronteiras existentes entre essas duas performances organizacionais.

São destacadas algumas limitações importantes deste estudo. Em primeiro lugar está o tamanho da amostra localizada em uma região restrita com características culturais e econômicas específicas. Também referente à amostra cita-se o fato de a mesma ser composta por uma grande variedade de empresas atuantes em variados segmentos industriais. Essas limitações prejudicam fortemente a capacidade de generalização das conclusões obtidas. Também se considera como limitação importante a ausência de indicadores econômicos e operacionais mais exatos, uma vez que os resultados desta pesquisa são baseados apenas na percepção dos empresários pesquisados. Sugerem-se, portanto, estudos com amostras maiores, localizadas em todos os estados brasileiros, assim como surveys focados em setores industriais específicos. Também se verifica a necessidade de estudos longitudinais, pois ao se considerar que os resultados de ações voltadas para o ambiente e para a sociedade tendem a trazer retornos econômicos para as empresas, em médio ou longo prazo, a investigação em uma linha de tempo mais abrangente pode vir a testar tais relações. 
Inovatividade e Performances Social, Ambiental e Econômica em MPMES: Uma Investigação Empírica

\section{REFERÊNCIAS}

Al-Tuwaijri, S. A., Christensen, T. E., Hughes, K. E. (2004). The relations among environmental disclosures environmental performance, and economic performance: a simultaneous equations approach. Accounting Organizations and Society, 29 (5-6), 447-471.

Baker, W. E., Sinkula, J. M. (1999). Learning orientation, market orientation and innovation: integrating and extending models of organizational performance. Journal of Market Focused Management, 4 (4), 295-308.

Baker, W. E., Sinkula, J. M. (2009). The complementary effects of market orientation and entrepreneurial orientation on profitability in small business. Journal of Small Business Management, 47 (4), 443-464.

Banerjee, S. B. (2003). Who sustains whose development? Sustainable development and the reinvention of nature. Organization Studies, 24 (1), 143-180.

BNDES (Banco Nacional de Desenvolvimento Econômico e Social). Classificação e perfil das MPEs brasileiras. 2012. Disponível em <http://www.bndes.gov.br/>.

Calantone, R., Garcia, R., \& Droge, C. (2003). The Effects of Environmental Turbulence on New Product Development Strategy Planning. Journal of Product Innovation Management, 20(2), 90-103. doi:10.1111/1540-5885.2002003

Carroll, A. (1999). Corporate social responsibility: evolution of a definitional construct. Business \& Society, 38 (3), 268-295.

Carter, C. R., Rogers, D. S. (2008). A framework of sustainable supply chain management: moving toward new theory. International Journal of Physical Distribution and Logistics Management, 39 (5), 360-387.

Cetindamar, D., Phaal, R., Prostre, D. (2009). Understanding Technology management as a dynamic capability: A framework for Technology management activities. Technovation, 29 (4), 237 246.

Ching-Hsun, C., Yu-Shan, C. (2013). Green organizational identity and green innovation. Management Decision, 51(5), 1056-1070.

Droge, C., Calantone, R., \& Harmancioglu, N. (2008). New Product Success: Is It Really Controllable by
Managers in Highly Turbulent Environments? Journal of Product Innovation Management, 25(3), 272-286. doi:10.1111/j.1540-5885.2008.00300.x

Elkington, J. (1998). Cannibals with forks: the triple bottom line of 21st century business, Stony Creek, CT: New Society Publishers.

FIERGS (Federação das Indústrias do Estado do Rio Grande do Sul). Cadastro Industrial do Rio Grande do Sul 2010. 2010. Disponível em <http://www.fiergs.com.br>.

Fornell, C., Larcker, D. (1982). Evaluating structural equation models with unobservable variables and measurement error. Journal of Marketing Research, 17 (1), 39-50.

Garrido, M., Camarero, C. (2010). Assessing the impact of organizational learning and innovation on performance in cultural organizations. International Journal of Nonprofit \& Voluntary Sector Marketing, 15(3), 215-232. doi:10.1002/nvsm.384

Gottlieb, D., Vigoda-Gadot, E., Haim, A., Kissinger, M. (2012). The ecological footprint as an educational tool for sustainability: a case study analysis in an Israeli public high school. International Journal of Educational Development, 32 (1), 193-200.

Gualandris, J., \& Kalchschmidt, M. (2014). Customer pressure and innovativeness: Their role in sustainable supply chain management. Journal of Purchasing and Supply Management, 20(2), 92103. doi:10.1016/j.pursup.2014.03.001

Guiral, A. (2012). Corporate Social Performance, Innovation Intensity, and Financial Performance: Evidence from Lending Decisions. Behavioral Research in Accounting, 24(2), 65-85.

Hair, J. F., Anderson, R. E., Tathan, R. L., Black, W. C. (1998). Multivariate data analysis. New Jersey: Prentice Hall.

Han, X., Hansen, E., Panwar, R., Hamner, R., \& Orozco, N. (2013). Connecting market orientation, learning orientation and corporate social responsibility implementation: is innovativeness a mediator? Scandinavian Journal of Forest Research, 28(8), 784-796. doi:10.1080/02827581.2013.833290

Hult, G. T. M., Hurley, R. F., Knight, G. A. (2004). Innovativeness: its antecedents and impact on business performance. Industrial Marketing Management, 33 (5), 429-438. 
Hurley, R. F., Hult, G. T. M. (1998). Innovation, market orientation and organizational learning: an integration and empirical examination. Journal of Marketing, 62 (3), 42-54.

IBGE (Instituto Brasileiro de Geografia e Estatística). Banco de dados agregados: IBGE automatic recovery system (SIDRA). 2009. Disponível em <http://www.ibge.gov.br>.

Knack, S., Keefer, P. (1997). Does social capital have an economic payoff? A cross-country investigation. Quarterly Journal of Economics, 112 (4), 12511288.

Kohli, A. K., Jaworski, B. J. (1990). Market orientation: the construct, research propositions, and managerial implications. Journal of Marketing, 54 (2), 1-18.

Li, Y. (2014). Environmental innovation practices and performance: moderating effect of resource commitment. Journal of Cleaner Production, 66, $450-458$.

Menon, A., Menon, A. (1997). Enviropreneurial marketing strategy: the emergence of corporate environmentalism as market strategy. Journal of Marketing, 61 (1), 51-57.

Miles, M. P., Covin, J. G. (2000). Environmental marketing: a source of reputational, competitive, and financial advantage. Journal of Business Ethics, 23 (3), 299-311.

Newell, P., Muro, A. (2006). Corporate Social and Environmental Responsibility in Argentina. Journal of Corporate Citizenship. 24, 49-68.

Orlitzky, M., Schmidt, F. L., Rynes, S. L. (2003). Corporate social and financial performance: a metaanalysis. Organization Studies, 24 (3), 403-441.

Paulraj, A. (2011). Understanding the relationships between internal resources and capabilities, sustainable supply management and organizational sustainability. Journal of Supply Chain Management, 47 (1), 19-37.

Perin, M. G., Sampaio, C. H., Hooley, G. (2007). Impacto dos recursos da empresa na performance de inovação. Rev. adm. empres. [online], 47 (1), 113.

Rao, P., Holt, D. (2005). Do green supply chains lead to competitiveness and economic performance? International Journal of Operations \& Production Management, 25 (9-10), 898-916.
Russo, M. V., Fouts, P. A. (1997). A resource-based perspective on corporate environmental performance and profitability. Academy of Management Journal, 40 (3), 534-559.

Schaefer, A. (2004). Corporate Sustainability Integrating Environmental and Social Concerns? Corporate Social Responsibility \& Environmental Management. 11 (4), 179-187.

SEBRAE (Serviço Brasileiro de Apoio às Micro e Pequenas Empresas). Critérios para a classificação de empresas. 2011. Disponível em <http://www.sebrae.com.br>.

Siguaw, J. A., Simpson, P. M., Enz, C. A. (2006). Conceptualizing innovation orientation: a framework for study and integration of innovation research. Journal of Product Innovation Management, 23 (6), 556-574.

Slaper, T. F., Hall, T. J. (2011). The Triple Bottom Line: What is it and how does it work? 2011. Disponível em < http://www.ibrc.indiana.edu/ibr/2011/spring/article 2.html >.

Tsai, K.-H., \& Yang, S.-Y. (2012). The contingent value of firm innovativeness for business performance under environmental turbulence. International Entrepreneurship and Management Journal, 10 (2), 343-366. doi:10.1007/s11365-0120225-4

Van den Bergh, J. C. J. M., Verbruggen, H. (1999). Spatial sustainability, trade and indicators: an evaluation the 'ecological footprint'. Ecological Economics, 29 (1), 61-72.

Wagner, M. (2005). How to Reconcile Environmental and Economic Performance to Improve Corporate Sustainability: Corporate Environmental Strategies in the European Paper Industry. Journal of Environmental Management. 76 (2), 105-118.

WCED (World Commission on Environmental and Development). (1987). Our Common Future. New York: Oxford University Press.

Webster, C. M., Gupta, S. (2005). Market orientation and innovation for strategic integration of exchange relationships: the case of argyle diamonds. In: ANZMAC Conference.

Zhou, K. Z., Yim, C. K., Tse, D. K. (2005). The effects of strategic orientations on technology- and marketbased breakthrough innovations. Journal of Marketing, 69 (2), 42-60. 